\title{
Stratégie de prospection hydrogéologique du socle de la bordure orientale tchadienne par optimisation du nombre et de la profondeur des sondages de reconnaissance Strategy of hydrogeological prospection in the eastern Chad basement by optimization of the number and depth of investigation boreholes
}

\section{P. Gombert}

Volume 12, numéro 3, 1999

URI : https://id.erudit.org/iderudit/705368ar

DOI : https://doi.org/10.7202/705368ar

Aller au sommaire du numéro

\section{Éditeur(s)}

Université du Québec - INRS-Eau, Terre et Environnement (INRS-ETE)

ISSN

0992-7158 (imprimé)

1718-8598 (numérique)

Découvrir la revue

Citer cet article

Gombert, P. (1999). Stratégie de prospection hydrogéologique du socle de la bordure orientale tchadienne par optimisation du nombre et de la profondeur des sondages de reconnaissance. Revue des sciences de l'eau / Journal of Water Science, 12(3), 597-608. https://doi.org/10.7202/705368ar

\section{Résumé de l'article}

Sous le climat à faible pluviosité du Tchad, les altérites sont dénoyées et seul le socle fracturé est aquifère. Le taux d'échec des forages atteint $60 \%$ car les fractures ont une répartition très discontinue comme le montre leur organisation fractale. Cela entraîne la coexistence de secteurs productifs et stériles. A l'échelle kilométrique, on peut ajuster le nombre de sondages de reconnaissance par village en fonction des caractéristiques climatiques, topographiques et géologiques. On définit ainsi des zones de productivité forte, où le taux de succès atteint $79 \%$, moyenne et faible. On peut alors affecter chaque village d'un " potentiel d'investigation " qui est le produit du nombre de sondages par leur profondeur prévisionnelle.

A l'échelle locale, une analyse en composantes principales des paramètres de forage montre que la présence d'eau souterraine est liée aux caractéristiques du socle fracturé et non altéré. Une analyse discriminante fournit une " équation de productivité " qui permet de prévoir $90 \%$ des résultats en cours de foration: dès que le forage a traversé une dizaine de mètres de socle non altéré, elle permet de définir une profondeur limite d'investigation dépendant des caractéristiques intrinsèques de chaque site. Elle est surtout applicable dans les zones les moins productives où l'on observe systématiquement un surcreusement inutile des forages négatifs.

On dispose ainsi d'une stratégie de prospection alliant le nombre et la profondeur des forages. Elle permet de limiter la profondeur des forages implantés sur des sites peu productifs et de reporter le métré ainsi récupéré sur des sites plus prometteurs. 


\title{
Stratégie de prospection hydrogéologique du socle de la bordure orientale tchadienne par optimisation du nombre et de la profondeur des sondages de reconnaissance
}

\author{
Strategy of hydrogeological prospection \\ in the eastern Chad basement by optimization \\ of the number and depth of investigation boreholes
}

\section{P. GOMBERT *}

Reçu le 4 novembre 1997, accepté le 21 juin 1999**.

\section{SUMMARY}

The aim of this study is to define a new strategy for groundwater prospection in sahelian basement aquifers. At present, the number and the depth of boreholes are fixed $a$ priori in the project document: these parameters are the same for all the villages, regardless of their environmental context. In fact, during the drilling campaign, we systematically observe a useless overdrilling of negative boreholes that affects the cumulative drilled length of the project (table 1). This is particularly important in granitic basement areas under the low rainfall sahelian climate: water is difficult to find because of low success rates, and the driller needs to ensure no groundwater indication appears a few meters under the fatal $60 \mathrm{~m}$ depth.

An illustration of this methodology is proposed for the Guéra, Ouaddai and Biltine provinces of eastern Chad (figure 1). This $150000 \mathrm{~km}^{2}$ area is situated at the border of the Chadian basin from 10 to $15^{\circ}$ north latitude at elevations of $400-700 \mathrm{~m}$, with an annual rainfall between 200 and $600 \mathrm{~mm}$. The geology is represented by precambrian granitoïds. Tectonics are well developed with many fractures, faults and photolineations from metric to multi-kilometric scales.

In Chad, weak recharge rates imply that the weathered rock reservoir is unsaturated and the aquifer is constituted by the fractured granitic basement. Thus, the overall success rate of 500 boreholes is only $42 \%$. The unequal distribution of fractures leads to the presence of productive and barren adjacent areas with significantly different success rates. A statistical analysis of photolineations shows their fractal distribution with a dimension around 1.57, similar to the 1.59 dimension obtained in fractal fracture models (figure 2). Fracturation is a main component of hydrogeological knowledge in basement

140, rue Auguste Renoir, 34500 Béziers, France.

* Correspondance. E-mail : philippe.gombert@hydroexpert.com

* * Les commentaires seront reçus jusqu'au 10 mai 2000. 
areas and its variations between the villages can explain the different potentials of basement productivity: we must consequently adjust an * investigation potential $\gg$ depending of the characteristics of each area. The proposed strategy of prospection determines the number of boreholes to drill and their specific depth.

At the kilometer scale, the total number of boreholes can be adjusted according to climatic, topographical and geological characteristics (table 2). We show that only four parameters can explain a range of success rate from 0 to $79 \%$ in different villages (table 3): altitude, average rainfall, petrography and fracturation intensity (measured in situ). Thus we can define the investigation potential which is the previous depth divided by the theoretical success rate of the area including the village. It is interesting to notice that the success rate in the high productivity class is similar to the average value obtained in more rainy basement countries of West Africa: for example $79 \%$ in south-west of Burkina Faso or $73 \%$ in Togo.

At the local scale, a principal components analysis on 12 drilling parameters was performed. It shows that the appearance of groundwater is mainly correlated to parameters describing the unweathered fractured rocks (figure 3). A discriminant analysis was then performed on four of these parameters: thickness of unweathered drilled basement, depth of first water arrival, number of water arrivals and hammer velocity in the unweathered basement. This yields a "productivity equation" which allows one to anticipate $90 \%$ of the borehole results (table 4). According to this equation, we can define a maximum investigation depth based on the geological characteristics of each borehole site.

The last section presents the complete strategy of groundwater basement prospection and two examples applied to Chad. For an average aquifer depth of $60 \mathrm{~m}$, the investigation potential of each village depends on its productivity class: it varies theoretically from 5 boreholes (i.e. $300 \mathrm{~m}$ ) in low productivity area to 1.3 boreholes (i.e. $76 \mathrm{~m}$ ) in high productivity zones (table 5). This potential must then be distributed among the different sites according to the result of their productivity equations.

The village of Getgéré is situated in a particularly unproductive zone (see table 3) where about ten boreholes are statistically needed to obtain a positive result: its investigation potential is supposed to be $300 \mathrm{~m}$. Four negative boreholes were drilled from 62 to $75 \mathrm{~m}$ with a total depth of $261 \mathrm{~m}$. In fact, the productivity equation showed all these sites were unproductive from drilled depths of 28 to $\mathbf{4 0} \mathrm{m}$ deep (table 6): the same result could have been obtained with only $130 \mathrm{~m}$ drilled; $131 \mathrm{~m}$ were uselessly consumed. With this excess drilled length, we could have drilled eight extra shallower boreholes and increased the probability of success in obtaining a productive well.

The village of Eroua is situated in a productive area where the success rate is $79 \%$ : its investigation potential is $60 / 0.79=76 \mathrm{~m}$. The first borehole was negative at depth of $74 \mathrm{~m}$, but the productivity equation already indicated this result after only $38 \mathrm{~m}$ of drilling. At the second site, a positive borehole was obtained at $41 \mathrm{~m}$ depth where the equation foresaw $43 \mathrm{~m}$. Finally, the cumulative drilled length was $115 \mathrm{~m}$ and the investigation strategy would have permitted the transfer of $34 \mathrm{~m}$ to another more promising site.

Key words: hydrogeology, basement, granite, borehole, success rate, statistical analysis, Africa. 


\section{1 - OBJECTIF}

L'hydraulique rurale vise à fournir de l'eau potable à des populations isolées à partir de forages généralement équipés de pompes à motricité humaine. La productivité ciblée est modeste et les forages sont le plus souvent déclarés positifs et équipés dès l'obtention d'un débit supérieur à $0,5 \mathrm{~m}^{3} \cdot \mathrm{h}^{-1}$ en fin de foration. Pour des raisons budgétaires, les profondeurs d'investigations sont contractuellement limitées, le plus souvent à $60 \mathrm{~m}$. Lorsqu'un sondage de reconnaissance n'atteint pas ce débit à la profondeur donnée, il est déclaré négatif et abandonné, puis le chantier se déplace sur un autre site. Malgré une présélection rigoureuse par photointerprétation et investigations géophysiques, tous les sites de sondage n'ont pas la même probabilité de succès. A l'heure actuelle, on ne peut prévoir leur productivité avant la foration et éliminer ainsi les sites les moins prometteurs : cela explique des taux d'échecs qui atteignent $60 \%$ dans les régions difficiles. En corollaire, les forages négatifs sont souvent surcreusés d'environ $20 \mathrm{~m}$ (tableau 1), ce qui correspond à un métré inutilement dépensé : il serait plus judicieux, dans les cas difficiles, de privilégier le nombre de forages plutôt que leur profondeur unitaire.

Tableau 1 Profondeur des forages implantés dans les granitoïdes de divers pays africains.

Table 1 Depth of boreholes in granitoids from different african countries.

\begin{tabular}{|c|c|c|c|c|c|}
\hline Localisation & $\begin{array}{c}\text { Effectif } \\
\text { de forages }\end{array}$ & \multicolumn{2}{|c|}{$\begin{array}{c}\text { Profondeur des forages } \\
\text { positits }\end{array}$} & $\begin{array}{c}\text { Taux } \\
\text { négatifs }\end{array}$ & Bibliographie succès \\
\hline Burkina Faso & 65 & $55 \mathrm{~m}$ & $79 \mathrm{~m}$ & $80 \%$ & Géohydraulique, 1989 \\
Burkina Faso & 199 & $42 \mathrm{~m}$ & $53 \mathrm{~m}$ & $76 \%$ & Gauff Ingenieure, 1991 \\
Tchad & 124 & $48 \mathrm{~m}$ & $62 \mathrm{~m}$ & $44 \%$ & Gombert, 1988 \\
Tchad & 156 & $60 \mathrm{~m}$ & $68 \mathrm{~m}$ & $42 \%$ & Wolf, 1990 \\
\hline
\end{tabular}

On propose ici une stratégie d'optimisation de la prospection hydrogéologique en deux phases: (1) présélection d'un nombre de forages adapté à chaque site puis (2) définition d'un critère d'arrêt de foration qui intègre les caractéristiques du terrain foré. Cette stratégie est illustrée par des exemples pris au Tchad où le taux moyen de succès n'est que de $44 \%$ au débit de $0,5 \mathrm{~m}^{3} \cdot \mathrm{h}^{-1}$.

\section{2 - PRÉSENTATION DE LA ZONE ÉTUdIÉE}

\subsection{Localisation}

La zone étudiée s'étend sur environ $150000 \mathrm{~km}^{2}$ le long de la bordure orientale du Tchad, entre 10 et $15^{\circ}$ de latitude Nord (figure 1). Les données proviennent de deux campagnes de forages, réalisées par le PNUD dans le cadre des 
projets CHD/85/004 (GOMBERT, 1988) et CHD/90/001 (WOLF, 1990), dans les préfectures du Biltine, du Ouaddaï et du Guéra. Le climat est tropical avec une pluviosité annuelle croissant de 200 à $800 \mathrm{~mm}$ du Nord au Sud.

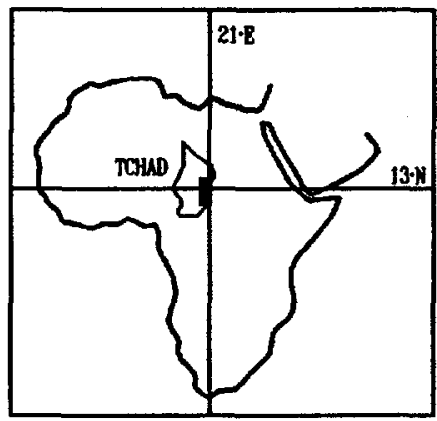

Figure 1 Localisation de la zone étudiée.

Location of studied area.

\subsection{Géologie}

La région appartient à un vaste batholite de granite calcoalcalin du Précambrien, appelé « granite régional » (PLOTTE, 1970), parcouru par de nombreuses intrusions : granitoïdes acides (granites alcalins) à basiques (granodiorites), filons d'aplite, de pegmatite et de quartz. Le socle est partout affleurant ou subaffleurant sous un mince recouvrement de dépôts quaternaires sablolimoneux issus des reliefs environnant. Par endroits se développe une couche d'altérites grenues, peu épaisse compte tenu de la faible efficience du climat actuel.

La tectonique cassante est présente mais irrégulièrement développée. A l'échelle plurikilométrique, l'analyse d'une carte de linéaments tracée par JOSEPH (1980) met en évidence le caractère fractal de leur distribution avec une dimension fractale de 1,57 (GOMBERT, 1993) : cette valeur est proche de celle de 1,59 donnée par le modèle de fragmentation de Turcotte (1992, voir figure 2). D'après MANDELBROT (1989), la présence de vastes zones vides est une caractéristique majeure des structures fractales ce qui, sur le plan tectonique, se traduit par la coexistence de secteurs intacts et fracturés.

\subsection{Hydrogéologie}

On retrouve ici la superposition classique d'un réservoir poreux superficiel (alluvions, colluvions et altérites) et d'un aquifère discontinu hébergé par les fractures du socle (CEFIGRE, 1990). La faiblesse et l'irrégularité des précipitations, jointe à l'importance de l'évapotranspiration, n'autorisent qu'une faible infiltration évaluée à $40 \mathrm{~mm}$ par an par MOUSSIE (1986). Ces conditions ne permettent pas de maintenir à saturation le réservoir aquifère superficiel (ENGALENC et al., 1981) et le niveau statique s'établit au sein même du socle fracturé. Cet aquifère discontinu est le reflet de la distribution fractale des fractures de la roche réservoir. Au Tchad, où l'altération argileuse est faiblement développée, le repérage des 


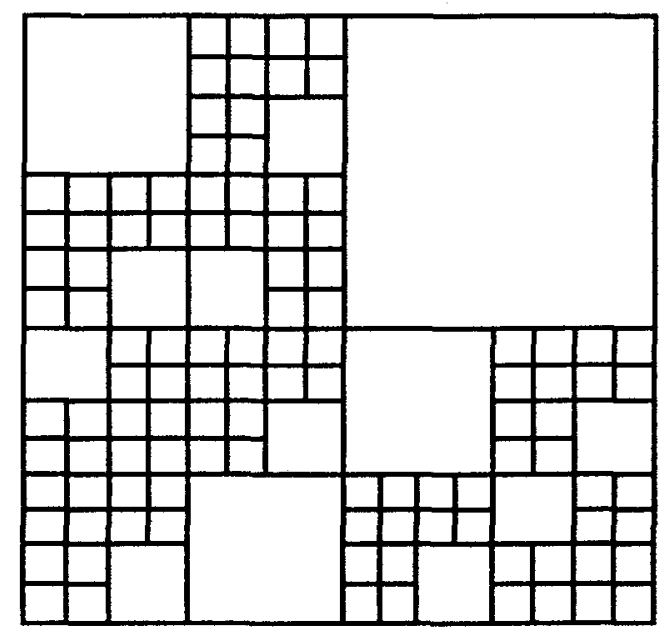

Figure 2 Modèle fractal de fragmentation de dimension 1,59 (in TURCOTTE, 1992). (les lignes de différentes longueurs simulent les fractures de différentes tailles)

Fractal model of fragmentation with dimension of 1.59 (in TURCOTTE, 1992).

(lines of different lengths represent the fractures of different sizes)

fractures aquifères est très peu sensible aux prospections géophysiques classiques, notamment géoélectriques (GOMBERT, 1992, 1997 et OUEDRAOGO, 1988) : l'étude de la fracturation joue alors un rôle fondamental (ASSOUMA et al., 1982).

\section{3 - ANALYSE DES FACTEURS DE PRODUCTIVITÉ}

\section{1 À l'échelle régionale}

Dans l'Ouaddaï et le Biltine, on a satisfait la demande en eau de 183 villages avec un taux de succès moyen des forages de $71 \%$. Parallèlement, une soixantaine de villages ont dû être abandonnés après au moins deux échecs successifs, totalisant ainsi 161 forages négatifs. GOMBERT (1988) a pu expliquer les causes d'une telle disparité grâce à une analyse multicritère portant sur les paramètres suivants : altitude du village, pluviosité moyenne, type de granitoïde subaffleurant et intensité de fracturation (tableau 2). Chaque paramètre est tout d'abord seuillé, puis sa valeur mesurée est remplacée par un " indice de productivité " qui vaut 0,1 ou 2 suivant son rôle dans la productivité des forages. En sommant les quatre indices de productivité obtenus par village, on définit des " classes de productivité " dont les taux de succès varient de 0 à $79 \%$ (tableau 3). Ces classes caractérisent des zones de productivité très différente et reflètent bien la discontinuité du réservoir aquifère à l'échelle plurikilométrique. II est intéressant de noter que le taux maximum de succès de $79 \%$ obtenu dans la classe la plus productive est comparable au taux moyen calculé pour les gra- 
nitoïdes d'Afrique de l'Ouest, soumis à des climats plus humides : $80 \%$ sur 457 forages au Burkina Faso (GEOHYDRAULIQUE-SOGREAH, 1989, GAUFF INGENIEURE, 1991 et DREAU, 1993), 73 \% sur 206 forages au Togo (ENGALENC, 1982).

Tableau 2 Attribution des valeurs de productivité aux villages étudiés (in GOMBERT, 1988).

Table 2 Attribution of productivity values to the studied villages (in GOMBERT, 1988).

\begin{tabular}{|l|c|c|c|}
\hline \multicolumn{1}{|c|}{ Paramètre } & Classes & Taux de succès & Indice de productivité \\
\hline Altitude & $<440 \mathrm{~m}$ & $25 \%$ & 0 \\
& $\geq 440 \mathrm{~m}$ & $52 \%$ & 1 \\
Pluviosité & $<425 \mathrm{~mm}$ & $29 \%$ & 0 \\
& $\geq 425 \mathrm{~mm}$ & $45 \%$ & 1 \\
Type de & granite alcalin & $56 \%$ & 2 \\
granitoide & granite calcoalcalin & $41 \%$ & 1 \\
& granodiorite & $13 \%$ & 0 \\
Intensité de & $<20$ fractures & $18 \%$ & 0 \\
fracturation & $38 \%$ & 1 \\
(sur cercle de $\left.300 \mathrm{~m}^{2}\right)$ & $\geq 40$ fractures & $56 \%$ & 2 \\
\hline
\end{tabular}

Tableau 3 Répartition du taux de succès par classe de productivité (in GOMBERT, 1988).

Table 3 Repartition of success rate in each productivity class (in GOMBERT, 1988).

\begin{tabular}{|l|c|c|c|c|}
\hline Classe de productivité & $\mathbf{0}$ à 1 & $\mathbf{2}$ à $\mathbf{4}$ & $\mathbf{5}$ à $\mathbf{6}$ & Non calculée \\
\hline Productivité & Nulle à faible & Moyenne & Forte & - \\
Effectif de sondages & 20 & 87 & 14 & 3 \\
Taux de succès & $0 \%$ & $41 \%$ & $79 \%$ & - \\
\hline
\end{tabular}

\section{2 À l'échelle locale}

Sur chaque forage, huit paramètres ont été mesurés : épaisseurs de recouvrement alluvio-colluvial (Rec), d'altérites (Alt) et de socle sain traversé (Soc), profondeur de la première venue d'eau (Pve) et nombre total de venues d'eau (Nve), nature pétrographique du socle (Nps), vitesse d'avancement dans les altérites (Vaa) et dans le socle sain (Vas). Un traitement par analyse en composantes principales montre que les paramètres représentatifs du socle sain (Soc et Vas) s'alignent le long de l'axe 1, tandis que ceux caractérisant les formations susjacentes (Rec, Alt et Vaa) s'opposent sur l'axe 2 (figure 3a). Les variables qui traduisent la présence d'eau souterraine (Pve et Nve) sont préférentiellement corrélées avec l'axe 1, confirmant que la productivité est liée aux caractéristiques du socle fracturé et non altéré. Dans le plan des individus, $90 \%$ des forages positifs possèdent une abscisse négative contre $18 \%$ des forages 
négatifs (figure $3 b$ ). Ceci va à l'encontre des résultats obtenus en Afrique de l'Ouest qui donnent aux altérites un rôle prépondérant dans la productivité des forages : cependant, dans le socle du nord du Burkina Faso, soumis à un climat comparable, OUEDRAOGO (1988) montre que l'épaisseur d'altérites peut devenir un facteur défavorable lorsqu'elle est trop importante.

a)

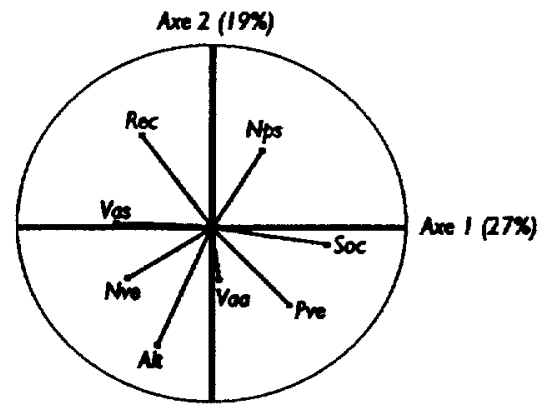

b)

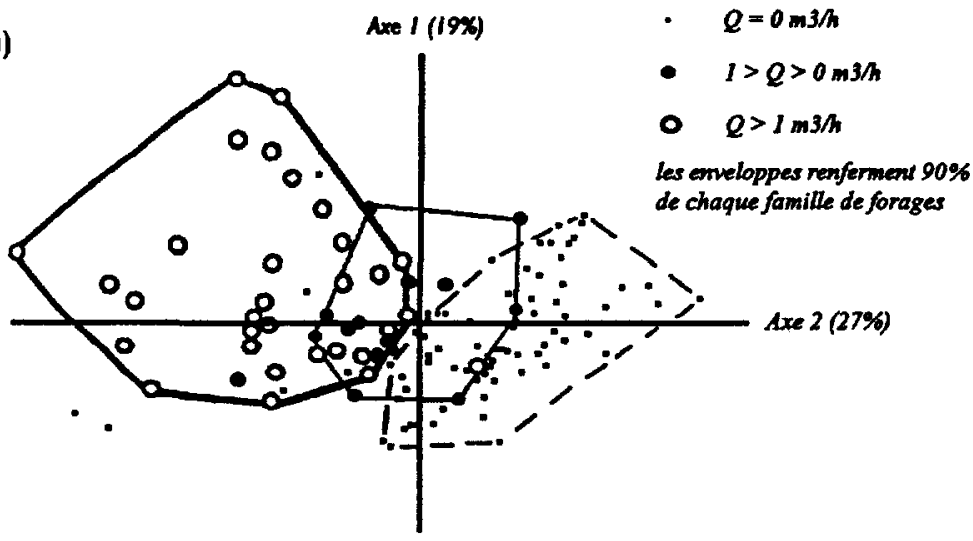

Figure 3 Analyse en composantes principales de données de forages (in GOMBERT, 1992).

a) dans l'espace des 8 paramètres ; b) dans l'espace des 124 forages

Principal components analysis of borehole data (in GOMBERT, 1992). a) In the space of parameters; $b$ ) in the space of boreholes

Une analyse discriminante a ensuite été effectuée avec la productivité $(X)$ comme variable expliquée et quatre paramètres représentatifs du socle sain et de la présence d'eau comme variables explicatives (Soc, Vas, Pve, Nve). En posant $X=100$ pour un forage positif et $X=0$ pour un forage négatif, on obtient une " équation de productivité " (1) qui permet de discriminer le résultat final des forages avec une marge d'erreur de l'ordre de $10 \%$ (GOMBERT, 1992) :

$$
X=141+7,89 \text { Vas }+1,32 \times 10^{2} \mathrm{Nve}+8,90 \text { Soc }-6,39 \text { Pve }
$$

Par définition, un forage est théoriquement positif dès lors que son potentiel de productivité à la profondeur considérée satisfait à $X \geq 50$. Cette équation montre que chaque site est en fait affecté d'une productivité de $X=141$ avant le 
début de la foration, tous les paramètres ayant alors une valeur nulle. Cette valeur traduit un fort potentiel initial de succès qui ne peut être lié qu'à la présélection opérée parmi les différents sites disponibles. En cours de foration, ce potentiel va fluctuer dans deux directions opposées :

- il augmente avec la vitesse d'avancement dans le socle sain, qui traduit l'état de fracturation de la roche non altérée, et avec le nombre de venues d'eau recoupées qui en est la conséquence hydrogéologique ;

- il diminue avec l'épaisseur de socle traversé et la profondeur de la première venue d'eau, paramètres contrôlant la profondeur du forage et, indirectement, celle du niveau statique.

L'équation (1) a été calculée d'après les résultats de 124 forages réalisés au Ouaddaî. Une validation de la méthode a consisté à appliquer cette équation à 118 forages du Guéra non pris en compte dans son calage (GOMBERT, 1992) : il en est résulté un classement correct de $90 \%$ des forages (tableau 4).

Tableau 4 Classement des forages par l'équation de productivité (in GOMBERT, 1992).

Table 4 Result of boreholes classification from productivity equation (in GOMBERT, 1992).

\begin{tabular}{|c|c|c|c|}
\hline Région & $\begin{array}{c}\text { Effectif } \\
\text { de forages }\end{array}$ & \multicolumn{2}{|c|}{$\begin{array}{l}\text { Forages bien classés } \\
\text { par l'équation } \\
\text { positifs négatifs }\end{array}$} \\
\hline Ouaddaĩ & 124 & $92 \%$ & $86 \%$ \\
\hline Guéra & 118 & $85 \%$ & $96 \%$ \\
\hline Total & 242 & $89 \%$ & $91 \%$ \\
\hline
\end{tabular}

L'équation (1) permet également de prévoir la profondeur limite théorique PIt à laquelle le potentiel de productivité va s'annuler, donc la probabilité de succès du forage à une profondeur donnée. II suffit de poser $X=0$, de considérer que les paramètres Vas et Nve resteront constants jusqu'à la profondeur finale et de remplacer le paramètre Soc par sa valeur en fonction de Plt obtenue dans l'équation (2):

$$
\mathrm{Plt}=\mathrm{Rec}+\mathrm{Alt}+\mathrm{Soc} \Rightarrow \mathrm{Soc}=\mathrm{Plt}-(\mathrm{Rec}+\mathrm{Alt})
$$

En résolvant les équations (1) et (2), on obtient:

- Pour Pve < Plt :

Plt $=1,58 \times 10^{1}+(\operatorname{Rec}+$ Alt $)+1,48 \times 10^{1}$ Nve $+8,87 \times 10^{1}$ Vas $-7,18 \times 10^{-1}$ Pve

- Pour Pve $\geq$ PIt, on décide que la profondeur de la première venue d'eau est, par défaut, celle du forage et l'on obtient alors :

$$
\text { PIt }=9,22+5,82 \times 10^{-1}(\operatorname{Rec}+\text { Alt })+8,63 \mathrm{Nve}+5,16 \times 10^{-1} \text { Vas }
$$

$\mathrm{Si}$ les paramètres Nve et Vas subissent des modifications en cours de foration, l'équation (3) ou (4) peut être recalculée en temps réel et fournir ainsi une profondeur limite théorique optimisée. 


\section{4 - OPTIMISATION DES PROFONDEURS DE FORATION}

\subsection{Calcul du potentiel d'investigation}

La stratégie adoptée pour la prospection débute par l'application d'un taux de succès moyen (Tsm) à chaque village en fonction de sa classe de productivité. On calcule ensuite un "potentiel d'investigation " (Pdi) basé sur la profondeur maximale contractuellement fixée par forage (Pmax) qui est généralement de $60 \mathrm{~m}$. Ce potentiel représente le métré théoriquement nécessaire pour obtenir un forage positif : selon la classe de productivité, il peut varier de 76 à $300 \mathrm{~m}$ (tableau 5). II se calcule de la manière suivante:

$$
\text { Pdi }=\text { Pmax } / \text { Tsm }=60 / \text { Tsm }
$$

Tableau 5 Calcul du potentiel d'investigation dans l'est du Tchad ("5 sondages de $60 \mathrm{~m})$.

Table 5 Calculation of the investigation potential in eastern Chad ("5 boreholes of $60 \mathrm{~m}$ ).

\begin{tabular}{|c|c|c|}
\hline Classe de productivité & Taux de succès attendu & Potentiel d'investigation \\
\hline Nulle à faible & $0 \%$ & estimé à $300 \mathrm{~m}^{*}$ \\
Moyenne & $41 \%$ & $146 \mathrm{~m}$ \\
Forte & $79 \%$ & $76 \mathrm{~m}$ \\
\hline
\end{tabular}

En cours de foration, on applique l'équation (1):

- si le résultat est négatif, le sondage de reconnaissance est arrêté et le métré résiduel reporté sur un autre site ;

- si le résultat est positif, le sondage est poursuivi jusqu'à la profondeur limite théorique indiquée par l'équation (3) ou (4), sans toutefois dépasser le potentiel d'investigation disponible.

La profondeur minimale à partir de laquelle l'équation de productivité peut être calculée correspond à la traversée de $10 \mathrm{~m}$ de socle non altéré. L'ordre de grandeur de cette épaisseur est suffisant pour obtenir une vitesse moyenne à l'avancement (Vas) : dans les granitoïdes du Burkina Faso, il est validé par le fait que les plus importantes venues d'eau surviennent généralement dans les vingt premiers mètres de socle sain où la densité de joints subhorizontaux et de diaclases est forte (BGR, 1987).

\subsection{Exemples d'application}

Un premier exemple concerne dans le village de Getgéré qui appartient à une classe de faible productivité. D'après le tableau 3 , seul un forage sur vingt est théoriquement positif, d'où l'intérêt de donner la priorité au nombre de forages plutôt qu'à leur profondeur. La volonté d'alimenter ce village en eau a conduit à la réalisation de quatre sondages de reconnaissance qui sont restés 
négatifs entre 62 et $75 \mathrm{~m}$ de profondeur. Grâce à l'équation (1), ce résultat était déjà prévisible à partir de 28 à $40 \mathrm{~m}$, la profondeur limite théorique ayant été dépassée (tableau 6). Dans ce village, où l'on disposait d'un potentiel global d'investigation de $300 \mathrm{~m}$, il aurait été plus judicieux de le convertir en une dizaine de forages de $30 \mathrm{~m}$ chacun avec approfondissement du plus prometteur. Cela aurait significativement augmenté les chances de succès sans dépassement du métré total, lequel a finalement atteint $261 \mathrm{~m}$ sans résultat positif.

Tableau 6 Profondeurs prévisionnelle et atteinte des forages négatifs du village de Getgéré.

(le potentiel de productivité est calculé à la * profondeur de calcul » correspondant à $10 \mathrm{~m}$ de pénétration dans le socle sain)

Table 6 Previous and final depths of negative boreholes in the village of Getgéré. (the productivity potential is calculated at the "calculation depth" corresponding to a $10 \mathrm{~m}$ penetration in the fractured basement)

\begin{tabular}{|l|c|c|c|c|c|}
\hline \multicolumn{1}{|c|}{ Forage } & \multicolumn{2}{|c|}{$\begin{array}{c}\text { Profondeur }(\mathbf{m}) \\
\text { limite } \\
\text { théorique }\end{array}$} & $\begin{array}{c}\text { Potentiel de } \\
\text { productivité }\end{array}$ & $\begin{array}{c}\text { Profondeur } \\
\text { atteinte }(\mathbf{m})\end{array}$ & $\begin{array}{c}\text { Métré } \\
\text { excédentaire }\end{array}$ \\
\hline Getgéré 1 & 28 & 28 & $-0,4$ & 75 & 47 \\
Getgéré 2 & 32 & 32 & $+0,0$ & 62 & 30 \\
Getgéré 3 & 34 & 31 & $-4,6$ & 62 & 31 \\
Getgéré 4 & 40 & 39 & $-1,1$ & 62 & 23 \\
Total & $\mathbf{1 3 4}$ & $\mathbf{1 3 0}$ & - & $\mathbf{2 6 1}$ & $\mathbf{1 3 1}$ \\
\hline
\end{tabular}

Le second exemple traite du village d'Eroua, classé en zone de forte productivité où deux sondages suffisent, en moyenne, pour obtenir un succès. Un premier sondage négatif a été arrêté à $74 \mathrm{~m}$ de profondeur alors que l'équation de productivité prévoyait ce résultat dès $38 \mathrm{~m}$. Le second forage s'est avéré positif à $41 \mathrm{~m}$ de profondeur : l'équation (1), calculée dès $30 \mathrm{~m}$, prévoyait effectivement une profondeur limite théorique de $43 \mathrm{~m}$. Sur cet exemple où l'on disposait d'un potentiel d'investigation de $76 \mathrm{~m}$, on aurait pu limiter la profondeur totale à $79 \mathrm{~m}$ au lieu des $115 \mathrm{~m}$ forés.

\section{5 - CONCLUSION}

Ce travail analyse la productivité des forages dans le socle de la bordure orientale tchadienne. À l'échelle régionale, les paramètres les plus influents sont de nature géomorphologique, climatologique, pétrographique et tectonique. Le classement des villages montre des zones contiguës de productivité faible, moyenne et forte. Une telle structure s'accorde avec ce que l'on sait de l'hydro- 
géologie régionale : dénoyage de l'aquifère continu des altérites et localisation de l'eau dans le réservoir discontinu formé par le socle fracturé. La coexistence de secteurs fracturés potentiellement aquifères et de secteurs non fracturés et stériles est en accord avec le caractère fractal de la fracturation, mis en évidence par l'étude des linéaments. À l'échelle locale, l'analyse multidimensionnelle des données de forage confirme que le socle fracturé est bien le principal réservoir aquifère. La probabilité de succès d'un forage peut alors être calculée en cours de foration, sitôt traversés les dix premiers mètres de socle non altéré, grâce à une équation de productivité : elle permet de définir une profondeur limite d'investigation à ne pas dépasser.

Afin de permettre la reconnaissance d'un maximum de sites sans augmenter le métré total de forage, on propose alors une stratégie de prospection qui privilégie le nombre de sites plutôt que leur profondeur. Son déroulement est le suivant :

- définition d'un " potentiel d'investigation " qui, en fonction de la classe de productivité du village, correspondra au métré cumulé de 1 à 5 forages de $60 \mathrm{~m}$ de profondeur ;

- foration sur un premier site avec arrêt du forage sitôt atteinte la profondeur limite théorique d'investigation calculée par l'équation de productivité ;

- report de l'excédent de métré sur un autre site plus prometteur jusqu'à atteindre le potentiel d'investigation accordé au village.

\section{RÉFÉRENCES BIBLIOGRAPHIQUES}

ASSOUMA D., BISCALDI R., LELONG F., MARTIN A., SAWADOGO J., SCANVIC J.Y., 1982. Recherches de critères d'implantation de forages hydrauliques productifs en formation de socle d'Afrique intertropicale. In : Les milieux discontinus en hydrogéologie, BRGM [Ed.], document $n^{\circ} 45$, pp. 25-32.

BARTON C.C., HSIEH P.A., 1989. Physical and hydrogeological properties of fractures. In : Proceedings of the 28th International Geological Congress, Am. Geophys.Union [Ed.], Field trip Guidebook T385.

BGR, 1987. Recherche sur la fracturation profonde en zone de socle cristallin à partir de forages à gros débit et de linéaments Landsat à l'aide de méthodes de géophysique avancées. Rapport BGR 99020 , Ouagadougou, Burkina Faso, Document CIEH, série Hydrogéologie, 129 p.

CEFIGRE, 1990. Synthèse des connaissances sur l'hydrogéologie de l'Afrique de l'Ouest. Socle cristallin et cristallophyllien. Sédimentaire ancien. Collection Maîtrise de l'Eau, 47 p., ministère de la Coopération et du Développement, Paris.

DREAU, 1993. Rapport final des travaux d'exécution des points d'eau de la quatrième phase du projet d'hydraulique villageoise de la boucle du Mouhoun. Direction Régionale de l'Eau du Mouhoun, ministère de l'Eau, Ouagadougou, Burkina Faso, $67 \mathrm{p}$.

ENGALENC M., 1982. Rôle de la photointerprétation dans la détermination des facteurs influençant la productivité des fractures du socle cristallin. In : Les milieux discontinus en hydrogéologie, BRGM [Ed.], document $n^{\circ} 45$, pp. 291-300.

ENGALENC M., GRILLOT J.C., LACHAUD J.C., 1981. Méthode d'étude et de recherche de l'eau souterraine des roches cristallines de l'Afrique de l'Ouest. GEOHYDRAULIQUE-LCHF [Ed.], Rapport en 3 volumes, Maisons-Alfort, France. 
GAUFF INGENIEURE, 1991. Assistance technique pour le projet d'hydraulique villageoise de la Comoé Phase III. Ministère de l'Eau, Ouagadougou, Burkina Faso, Rapport final, $71 \mathrm{p}$

GÉOHYDRAULIQUE-SOGREAH, 1989. Assistance technique pour le programme d'hydraulique villageoise dans les provinces du Houet et du Kénédougou. Ministère de l'Eau, Ouagadougou, Burkina Faso, Rapport final, 252 p.

GOMBERT P., 1988. Synthèse des connaissances acquises par le projet CHD/85/004 sur l'hydrogéologie du Ouaddaï, Tchad. PNUD-DTCD, Projet CHD/85/004, N'Djaména, Tchad. Rapport 13/HG/88, $47 \mathrm{p}$.

GOMBERT P., 1990. Analyse des facteurs de productivité des sondages et optimisation de la profondeur d'investigation dans la préfecture du Ouaddaï (Tchad). Séminaire sur l'optimisation des méthodes de recherche et d'exploitation des eaux souterraines dans les régions de socle sahélien à faible pluviosité, PNUD-DTCD, N'Djaména, Tchad, avril 1990, 7 p.

GOMBERT P., 1992. Éléments d'appréciation de la présence d'eau souterraine dans le socle de la bordure orientale tchadienne. Actes du Colloque international sur l'hydrogéologie des milieux discontinus sous climat aride, Faculté des Sciences Semlalia, Marrakech, Maroc, avril 1992, 123-126.

GOMBERT P., 1993. Analyse fractale et multifactorielle du socle sahélien en bordure de la cuvette tchadienne. Actes des $2^{e}$ Journées Tunisiennes de Géologie Appliquée, ENIS, Sfax, Tunisie, 17-19 mai 1993, 11-19.
GOMBERT P., 1997. Variabilité spatiale de la productivité aquifère du socle sahélien en hydraulique rurale. In : Hard Rock Hydrosystems, POINTET T. [Ed.], IAHS Publ. $n^{\circ} 241,113-122$

JOSEPH G., 1980. L'hydrogéologie du Ouaddaï dans le contexte géologique et hydroclimatique de la république du Tchad. Th. $3^{e}$ cycle, Univ. Nice, France, $197 \mathrm{p}$.

MANDELBROT B., 1989. Les objets fractals. FLAMMARION, Paris

MOUSSIE B., (1986). Carte de potentialités des ressources en eau souterraine de l'Afrique occidentale et centrale au 1/5 000000 . BRGM, Orléans, France Rapport 86 AFO 178 EAU, 82 p.

OUÉDRAOGO B., 1988. Productivité des forages sur socle cristallin et cristallophyllien en région subsahélienne. Th. Doct., Univ. Le Mans, France, 252 p.

PLOTTE H., 1970. Hydrogéologie de l'Ouaddaĩ. Programme quadriennal d'aménagement hydraulique de l'Ouaddaï. BRGM, Orléans, France, Rapport 70 YAO 004 LAM, 423 p.

TURCOTTE D.L., 1992. Fractals and chaos in geology and geophysics. Cambridge University Press, Cambridge, UK.

WOLF M.H., 1990. Résumé des résultats des travaux de forage effectués dans la préfecture du Guéra (Tchad). Séminaire sur l'optimisation des méthodes de recherche et d'exploitation des eaux souterraines dans les régions de socle sahélien à faible pluviosité, PNUD-DTCD N'Djaména, Tchad, avril 1990, 9 p. 